\begin{tabular}{|c|l|}
\hline Title & $\begin{array}{l}\text { Biochemical and Immunochemical Characterization of Two Discrete V itellogenin Proteins and Their Derived } \\
\text { Lipovitellins in the Inshore Hagfish (Eptatretus burgeri) }\end{array}$ \\
\hline Author(s) & Nishimiya, Osamu; Kunihiro, Y Ysuy uki; Hiramatsu, Naoshi; Inagawa, Hiroy uki; Todo, Takashi; Hara, A kihiko \\
\hline Citation & $\begin{array}{l}\text { Zoological Science, 31(4), 251-257 } \\
\text { https:/doi.org/10.2108/2s130234 }\end{array}$ \\
\hline Issue Date & 201404 \\
\hline Doc URL & http://hdl.handle.net/2115/58267 \\
\hline Type & article \\
\hline File Information & zs130234.pdf \\
\hline
\end{tabular}

Instructions for use 


\title{
Biochemical and Immunochemical Characterization of Two Discrete Vitellogenin Proteins and Their Derived Lipovitellins in the Inshore Hagfish (Eptatretus burgeri)
}

\author{
Osamu Nishimiya ${ }^{1}$, Yasuyuki Kunihiro ${ }^{1}$, Naoshi Hiramatsu ${ }^{1 *}$, \\ Hiroyuki Inagawa ${ }^{2}$, Takashi Todo ${ }^{1}$, and Akihiko Hara ${ }^{1}$ \\ ${ }^{1}$ Faculty of Fisheries Sciences, Hokkaido University, Hakodate, Hokkaido 041-8611, Japan \\ ${ }^{2}$ Department of Applied Aquabiology, National Fisheries University, \\ Shimonoseki, Yamaguchi 759-6595, Japan
}

\begin{abstract}
Vitellogenesis has been extensively studied in oviparous vertebrates, including teleost fishes, while not much is known with regard to jawless hagfishes, modern representatives of the most primitive vertebrate class. This study aimed to characterize vitellogenin (Vtg) and yolk protein (YP) in the inshore hagfish (Eptatretus burgeri) as an initial step to understand vitellogenesis in this species. A putative Vtg fraction was purified from the serum of female hagfish by combinations of hydroxylapatite and ion-exchange chromatography, followed by gel filtration. The purified fraction appeared to contain two distinct Vtgs (Vtg1 and Vtg2) and exhibited biochemical properties resembling those previously reported for teleost Vtgs; these appeared to be female-specific serum proteins and high-molecular-weight proteins in gel filtration $(\sim 505 \mathrm{kDa}$ as the mixture fraction of both Vtgs) and in SDS-PAGE (Vtg1 and Vtg2; $210 \mathrm{kDa}$ and $\sim 195 \mathrm{kDa}$, respectively). A major YP was also purified from hagfish eggs by combinations of hydroxylapatite chromatography and gel filtration; the apparent native mass of the purified YP was unusually large (>669 kDa). The purified YP consisted of four polypeptides in SDS-PAGE; the peptide pattern indicated that it consisted of two lipovitellins (Lv1 and Lv2) giving rise to two sets of heavy chains ( 116 kDa and $\sim 106 \mathrm{kDa}$, respectively) and two light chains ( $\sim 32 \mathrm{kDa}$ and $\sim 28 \mathrm{kDa}$, respectively). Additional immunological analysis, $\mathrm{N}$ terminal amino acid sequencing and cDNA cloning firmly confirmed the precursor-product relationship between hagfish Vtgs and Lvs.
\end{abstract}

Key words: Agnatha, Eptatretus burgeri, lipovitellin, vitellogenin, yolk protein

\section{INTRODUCTION}

Vitellogenin $(\mathrm{Vtg})$ is a major precursor of egg yolk proteins (YPs) in oviparous vertebrates. Following its synthesis by the liver of maturing females in response to estradiol-17 $\beta$ (E2), Vtg is secreted into the bloodstream and incorporated into growing oocytes by receptor-mediated endocytosis (Wallace, 1985; Speaker and Sullivan, 1994). Prior to its deposition in yolk granules, Vtg undergoes limited proteolytic cleavage, giving rise to several classes of YPs. In teleosts, Vtg-derived YPs are typically designated as lipovitellin (Lv), phosvitin (Pv) and $\beta^{\prime}$-component ( $\beta^{\prime}$-c) (Matsubara and Sawano, 1995; Hiramatsu and Hara, 1996; Hiramatsu et al., 2002b, c), while some other YP variants (e.g., Cterminal peptide and $\mathrm{Lv}-\mathrm{Pv}$ complexes) are also evident (Hiramatsu et al., 2005, 2006; Sawaguchi et al., 2005; Amano et al., 2007b).

\footnotetext{
* Corresponding author. Tel. : : +81-138-40-8878;

Fax : +81-138-40-8878;

E-mail: naoshi@fish.hokudai.ac.jp
}

doi:10.2108/zs130234
In recent years, a new "multiple Vtg model" has been proposed to describe vitellogenesis in teleosts based on the structural diversity of Vtg (Hiramatsu et al., 2005, 2006; Finn and Kristoffersen, 2007; Reading et al., 2009); typically, three kinds of vtg cDNA (i.e., vtgAa, vtgAb, and $v \operatorname{tg} C$ ) are found in a variety of highly evolved teleosts. Of the teleosts presenting three vtg transcripts, all corresponding Vtg proteins (VtgAa, VtgAb, and VtgC) have been identified in some species, including white perch, Morone americana (Hiramatsu et al., 2002c), mosquitofish, Gambusia affinis (Sawaguchi et al., 2005), red seabream, Pagrus major (Sawaguchi et al., 2006), and grey mullet, Mugil cephalus (Amano et al., 2007a).

The concept of the multiple Vtg model remains to be tested in primitive oviparous vertebrates that are more basal than teleosts. Agnatha, the most primitive vertebrate clade, consists of Petromyzonids (lamprey) and Myxinoids (hagfish). These have been recognized as the most important animals for understanding the evolution of vertebrates (Hall, 1998, 1999). Some studies on Vtg and/or YPs have been conducted in these species in order to understand evolutionary aspects of vertebrate vitellogenesis. For example, Meininger 
et al. (1984) reported the crystal structure of Lv in the silver lamprey (Ichthyomyzon unicuspis) and the sea lamprey (Petromyzon marinus). Subsequently, Raag et al. (1988) analyzed the crystal structure of the lipid-protein complex (Lv-Pv) in the silver lamprey. Moreover, Sharrock et al. (1992) reported the primary structure of Vtg and verified that LV is a component of Vtg in the silver lamprey. Mewes et al. (2002) demonstrated that Vtg protein was detectable in the serum of male European river lamprey (Lampetra fluviatilis) treated with high concentrations of E2, indicating that E2 is involved in the induction and regulation of $\mathrm{Vtg}$ synthesis. Subsequently, Vtg was isolated from the blood of the maturing female river lamprey.

Yu et al. (1980) immunologically detected a yolk precursor protein using antiserum against $Y P$ in the serum of the vitellogenic female Pacific hagfish (Eptatretus stouti). Moreover, Yu et al. (1981) showed that plasma Vtg was rich in phosphate and induced by E2 treatment in male and immature Pacific hagfish. In addition, Lange and Richer (1981) demonstrated that the crystal structure of Lv in the Atlantic hagfish (Myxine glutinosa) is similar to that in African clawed frog (Xenopus laevis). As opposed to lampreys, however, characterization of $\mathrm{Vtg}$ and YP has not, to date, been completed in hagfish.

Since most hagfish species inhabit deep marine environments, studies on their reproductive physiology have been undertaken to a much lesser extent than in lamprey species. The inshore hagfish, Eptatretus burgeri, can live close to the coast. Moreover, this species has a wider range of temperature and salinity tolerance compared to other hagfish species distributed around Japanese coasts (Ota and Kuratani, 2006), resulting in the frequent use of this species in research.

The objective of this study is to characterize Vtg and Vtg-related YP in the inshore hagfish in order to obtain a basis to understand the mechanisms underlying vitellogenesis of this most primitive egg laying vertebrate. To achieve this, we specifically aimed to: (1) detect and purify Vtg and YP using immunological and biochemical techniques, and (2) examine their relationship.

\section{MATERIAL AND METHODS}

\section{Experimental animals and tissue sample}

Specimens of inshore hagfish, caught from coastal aquatic areas around Futaoi Island, Yamaguchi Prefecture, Japan, were purchased from a local fisherman. These were transported by air to the Faculty of Fisheries Sciences, Hokkaido University, and sampled immediately after arrival. All experimental procedures involving live fish followed the policies and guidelines of the Hokkaido University Animal Care and Use Committee.

After anesthetizing the animals in FA-100 (Mitsubishi Tanabe Pharma, Osaka, Japan), their blood and tissues, including ovary and liver, were taken. Following incubation at $4{ }^{\circ} \mathrm{C}$ overnight, the blood samples were centrifuged at $10,000 \mathrm{rpm}$ for $10 \mathrm{~min}$. The resulting serum aliquots were stored at $-80^{\circ} \mathrm{C}$ until use.

The yolk samples were obtained from fully-grown oocytes by syringe and mixed with 10 volumes of $0.02 \mathrm{M}$ Tris- $\mathrm{HCl}, \mathrm{pH} 8.0$ containing $1 \mathrm{M} \mathrm{NaCl}$. The yolk samples were centrifuged for $10 \mathrm{~min}$ at $10,000 \mathrm{rpm}$. The resulting supernatant was collected as yolk extracts (YE) and stored at $-80^{\circ} \mathrm{C}$ until use. Liver samples were cut into small pieces and fixed in RNAlater (Ambion, Austin, TX, USA). After incubation at $4^{\circ} \mathrm{C}$ overnight, liver samples were stored at $-80^{\circ} \mathrm{C}$ until use as a source of total RNA for cDNA cloning of hagfish vtg genes.

\section{Antisera}

Polyclonal antisera were raised in rabbits against hagfish male serum (anti-Male), female serum (anti-Female), yolk extracts (antiYE), purified Vtg (anti-Vtg), and purified LV (anti-LV) by intradermal injection of each antigen emulsified with an equal volume of Freund's complete adjuvant (Merck, Darmstadt, Germany).

Preparation of all antisera was performed according to the methods described in our previous reports (Amano et al., 2007a, b) using an intradermal administration protocol $(0.25 \mathrm{mg}$ antigen per injection $\times$ four times). Antisera were pre-absorbed with an equal volume of male serum to obtain absorbed anti-Female serum (ab. anti-Female) and absorbed anti-YE serum (ab. anti-YE) for the detection of Vtgs and Vtg-related YPs. Antisera were stored at $-30^{\circ} \mathrm{C}$ until use.

\section{Column chromatography}

Column chromatography using Fast Flow Type hydroxylapatite (HA) media (Nacalai Tesque, Kyoto, Japan) was performed for the initial step of Vtg and YP purification. The HA medium was loaded into a $2.5 \times 8 \mathrm{~cm}$ glass column (Bio-Rad, Hercules, CA, USA) and equilibrated with an appropriate starting buffer: $0.1 \mathrm{M}$ potassium phosphate (KP), $\mathrm{pH} 6.8$, for $\mathrm{Vtg}$ purification and $0.1 \mathrm{M} \mathrm{KP}, \mathrm{pH} 6.8$, containing $1 \mathrm{M} \mathrm{NaCl}$ for $\mathrm{YP}$ purification. Samples were eluted by step-wise additions of various concentration of KP $(0.1 \mathrm{M}-1.2 \mathrm{M} \mathrm{KP}$, see elution profiles in figures) at a flow rate of $60 \mathrm{ml} / \mathrm{h}$. Eluted fractions were collected in a volume of $1.5 \mathrm{ml}$ per tube.

Anion-exchange chromatography was performed using a POROS perfusion chromatography medium (POROS $50 \mathrm{HQ}$; Applied Biosystems, Foster City, CA, USA). The POROS $50 \mathrm{HQ}$ medium was loaded into a $1 \mathrm{~cm} \times 30 \mathrm{~cm}$ column and fitted to a fast protein liquid chromatography (FPLC) system (GE Healthcare, Buckinghamshire, England). Samples were loaded onto the column equilibrated with $0.02 \mathrm{M}$ Tris- $\mathrm{HCl}(\mathrm{pH} 9.0)$ containing $0.15 \mathrm{M} \mathrm{NaCl}$ and subsequently eluted by a gradient addition of $\mathrm{NaCl}(0.15 \mathrm{M}$ to $0.6 \mathrm{M}$ ) to the Tris- $\mathrm{HCl}$ buffer at a flow rate of $2 \mathrm{ml} / \mathrm{min}$. Eluted fractions were collected at a volume of $1 \mathrm{ml}$ per tube.

Gel filtration using a Superose 6 prep grade (GE Healthcare; $1 \mathrm{~cm} \times 30 \mathrm{~cm}$ ) was performed for the purification of Vtgs and YPs. Samples were typically eluted with either $0.02 \mathrm{M}$ Tris- $\mathrm{HCl}$ buffer $(\mathrm{pH}$ 8.0) containing $2 \% \mathrm{NaCl}$ and $0.1 \% \mathrm{NaN}_{3}$ for the purification of $\mathrm{Vtgs}$ or $0.02 \mathrm{M}$ Tris- $\mathrm{HCl}$ buffer (pH 8.0) containing $1 \mathrm{M} \mathrm{NaCl}$ and $0.1 \%$ $\mathrm{NaN}_{3}$ for the purification of YPs. The column flow rate was $0.5 \mathrm{ml} / \mathrm{min}$ and fractions were collected at a volume of $0.5 \mathrm{ml}$ per tube. Ferritin (440 kDa) and thyroglobulin (669 kDa) were used as marker proteins to estimate the relative molecular masses of $\mathrm{Vtg}$ in the gel filtration.

Eluted fractions obtained during the column chromatography were monitored for absorbance at $280 \mathrm{~nm}\left(\mathrm{OD}_{280}\right)$.

\section{Electrophoresis and $\mathrm{N}$-terminal amino acid sequence}

Electrophoresis and $\mathrm{N}$-terminal amino acid sequencing were performed following the method described in Amano et al. (2007b). Simply, after trans-blotting following SDS-PAGE, peptide bands were visualized on the polyvinylidene difluoride (PVDF) membrane (Immobilon-PSQ; Millipore, Bedford, MA, USA) by staining with Coomassie Brilliant Blue (CBB), cut out from the membrane and subjected to N-terminal amino acid sequencing on a Model 492 Procise Sequencing System (Applied Biosystems, Foster City, CA, USA).

Isolation of partial cDNA clones encoding hagfish vitellogenins

All molecular biological procedures described below were performed according to the manufacturer's protocol unless otherwise stated. Total RNA was extracted from the liver of vitellogenic female hagfish using ISOGEN (Nippon-GENE, Tokyo, Japan). The cDNA 
template for $5^{\prime}$ - rapid amplification of CDNA ends (5'-RACE) was prepared from the hepatic total RNA using SMART RACE CDNA Amplification Kit (Clontech, Mountain View, CA, USA). Primers used in the $5^{\prime}$-RACE of Vtgs were designed based on partial sequences of inshore hagfish vtg1 and vtg2 (Nishimiya et al., manuscript in preparation); Vtg1: 5'-TGATACCAGCCCGTGCGAGACCG-3' and Vtg2: 5' - CAGACGGGGGTCTTTGCACAGAAGCGG-3'. Each PCR product amplified by the $5^{\prime}$-RACE procedure was separated by $1.5 \%$ agarose gel electrophoresis and purified using QIAquick Gel Extraction Kit (QIAGEN; Hilden, Germany). The extracted products were ligated into pGEM-T Easy vector (Promega, Madison, WI, USA) and transformed into XL1-blue E. coli. Positive clones were selected and subcultured for the extraction of plasmid DNA using Wizard Plus SV Minipreps DNA Purification system (Promega). Plasmid DNAs containing Vtg insert were sequenced using BigDye Terminator v3.1 Cycle Sequencing kit (Applied Biosystems, Carlsbad, CA, USA) and the Applied Biosystems 3130xl Genetic Analyzer (Applied Biosystems).

\section{RESULTS}

\section{Detection of vitellogenin and yolk proteins}

Male serum, female serum and YE were subjected to SDS-PAGE and subsequently analyzed by Western blotting using ab.anti-YE (Fig. 1). In SDS-PAGE, two bands with apparent molecular masses of $\sim 210$ and $\sim 195 \mathrm{kDa}$ were observed in female, but not in male, serum; these femalespecific high molecular weight polypeptides were considered to be putative Vtgs in the hagfish. Yolk extracts consisted of several polypeptide bands including two major bands with high molecular weights ( 116 and $\sim 106 \mathrm{kDa})$, which were identified as putative Lv polypeptides due to their size. Western blotting using ab.anti-YE recognized two putative Vtg polypeptides in female serum, while it faintly recognized the $\sim 106 \mathrm{kDa}$ putative Lv band in YE besides many smaller YP polypeptides.

\section{Purification of vitellogenin}

For the purification of inshore hagfish Vtg, serum obtained from a vitellogenic female hagfish was initially applied to HA column chromatography. Elution was performed by step-wise addition of KP buffer (5 steps; see Fig. 2A). When the detection of Vtg was performed using the ab.anti-YE, fractions eluted at the $0.8 \mathrm{M} \mathrm{KP}$ step appeared to contain female-specific YPrelated proteins (i.e., putative Vtgs). These putative Vtg fractions were pooled and dialyzed against $0.02 \mathrm{M}$ Tris- $\mathrm{HCl}$ buffer $(\mathrm{pH}$ 9.0) containing $0.15 \mathrm{M} \mathrm{NaCl}$. The pooled fraction was subjected to anion-exchange chromatography on POROS $50 \mathrm{HQ}$ column. A gradient addition of $\mathrm{NaCl}(0.15 \mathrm{M}$ to $0.6 \mathrm{M})$ was used to further fractionate $\mathrm{Vtg}$ from other serum proteins (Fig. 2B). Immunoreactive fractions eluted from the POROS column were pooled and dialyzed against $0.02 \mathrm{M}$ Tris- $\mathrm{HCl}$ buffer $(\mathrm{pH}$ 8.0) containing $2 \% \mathrm{NaCl}$ and $0.1 \% \mathrm{NaN}_{3}$, and the pooled

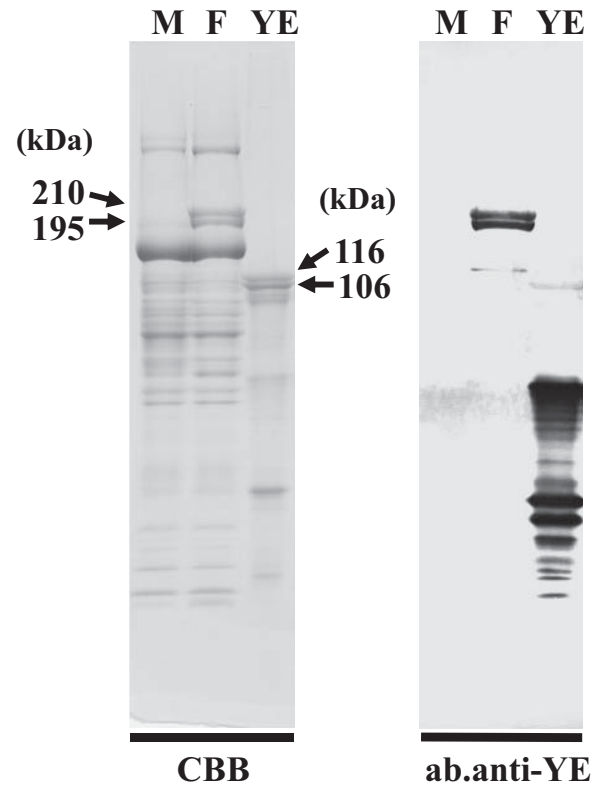

Fig. 1. 5-22.5\% SDS-PAGE and corresponding Western blotting of male serum (M), female serum (F) and yolk extracts (YE). Antisera used in Western blotting were raised in rabbit against $Y E$ extracts, which were pre-absorbed with male serum (ab.anti-YE). CBB: Coomassie Brilliant Blue staining.
A

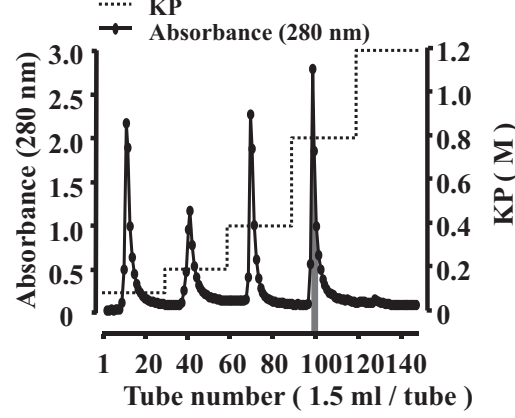

C

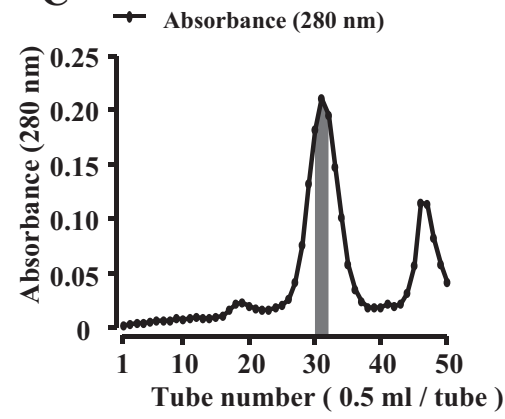

B

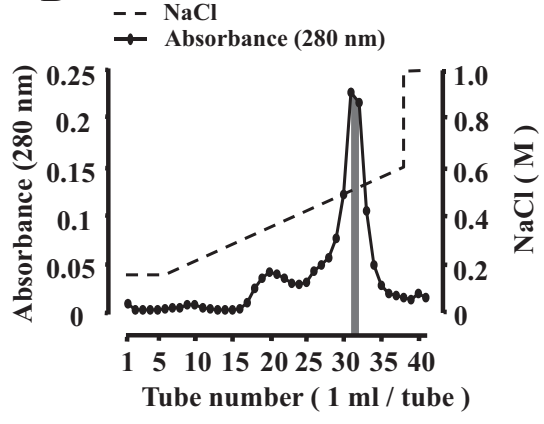

D

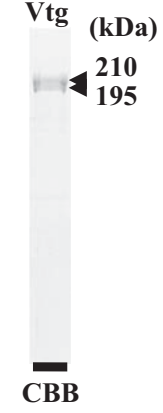

Fig. 2. Elution patterns of chromatography on hydroxylapatite column (A) followed by chromatography on POROS $50 \mathrm{HQ}$ column (B) and gel filtration on Superose 6 column (C) during the purification of the hagfish vitellogenin (Vtg). The shaded area in chromatogram $A$ indicates fractions eluted by addition of $0.8 \mathrm{M}$ potassium-phosphate (KP) buffer (i.e., Vtg-rich fraction). The $0.8 \mathrm{M} \mathrm{KP}$ fractions were pooled and applied onto the POROS $50 \mathrm{HQ}$ column; the shaded area eluted in this column indicates fractions pooled as a crude putative $\mathrm{Vtg}$ fraction. Finally, this crude $\mathrm{Vtg}$ was applied onto Superose 6 column; the shaded fractions eluted in this column were collected as purified putative $\mathrm{Vtg}$ in the hagfish. (D) $7.5 \%$ SDS-PAGE of purified putative $\mathrm{Vtg}$. The SDSPAGE gel was stained with Coomassie Brilliant Blue (CBB). 
fraction was subjected to the gel filtration on Superose 6 prep grade column. The chromatogram obtained in the gel filtration revealed a major, symmetrical peak containing the putative Vtg peptides at a position corresponding to $\sim 505 \mathrm{kDa}$, as well as two other minor peaks (Fig. 2C). Two bands ( 210 and $195 \mathrm{kDa})$ were observed when the major $\sim 505 \mathrm{kDa}$ peak was subjected to SDS-PAGE (Fig. 2D).

\section{Purification of lipovitellin}

For the separation of putative Lv, YE were initially dialyzed against $0.1 \mathrm{M} \mathrm{KP}$ buffer ( $\mathrm{pH}$ 6.8) containing $1 \mathrm{M} \mathrm{NaCl}$ to be used as the starting material.

Elution of the samples from the HA column was performed by step-wise addition of KP buffer ( 5 steps; see Fig. $3 A)$. When the detection of putative Lv was performed by the combination of SDS-PAGE and immunological procedures using ab.anti-YE, fractions eluted at the 0.4 and 0.8 M KP steps appeared to contain $\sim 116$ and 106 kDa peptides representing putative Lv (data not shown). Because $0.8 \mathrm{M} \mathrm{KP}$ fractions predominantly contained putative Lv, these fractions were pooled and dialyzed against $0.02 \mathrm{M}$ Tris- $\mathrm{HCl}$ buffer $(\mathrm{pH} 8.0)$ containing $1 \mathrm{M} \mathrm{NaCl}$. Gel filtration of the pooled putative Lv fraction on Superose 6 prep grade column revealed a symmetrical peak at a position corresponding to $>669 \mathrm{kDa}$ (Fig. 3B). Fractions eluted around this peak were collected and pooled as purified putative Lvs. Two main bands $(\sim 116$ and $\sim 106 \mathrm{kDa})$ and minor bands ( $\sim 32$ and $\sim 28 \mathrm{kDa}$ ) were observed when the > $669 \mathrm{kDa}$ peak was subjected to SDS-PAGE (Fig. 3C).

\section{Immunobiochemical relations between vitellogenin and yolk proteins}

Polyclonal antisera were raised in rabbits against purified products (anti-Vtg and anti-Lv). Specificity of these antisera was tested by Western blotting (Fig. 4). The anti-Vtg recognized two polypeptide bands ( 210 and $\sim 195 \mathrm{kDa}$ putative Vtgs) in female serum, but did not recognize any proteins in male serum, indicating that anti-Vtg was specific to the putative Vtg antigens. The anti-Lv exhibited the same immunoreactive pattern when Western blotting was conducted for sera of female and male. On the other hand, antiVtg appeared to react with YE exhibiting three main bands with relative masses of $\sim 116, \sim 32$ and $\sim 28 \mathrm{kDa}$, as well as multiple bands with apparent masses ranging from $\sim 62$ to $\sim 43 \mathrm{kDa}$. The anti-Lv recognized three main bands with relative masses of $\sim 116, \sim 106$, and $\sim 32$, as well as multiple bands with apparent masses ranging from $\sim 80$ to $\sim 28 \mathrm{kDa}$.

\section{$\mathrm{N}$-terminal amino acid sequence analysis}

Polypeptides of putative Vtgs, namely bands with masses of $\sim 210 \mathrm{kDa}(210 \mathrm{Vtg})$ and $\sim 195 \mathrm{kDa}(195 \mathrm{Vtg})$, were analyzed for determining their $\mathrm{N}$-terminal amino acid sequences. The same analyses were performed on the putative heavy $(\mathrm{H})$ chains of Lv, namely bands with masses

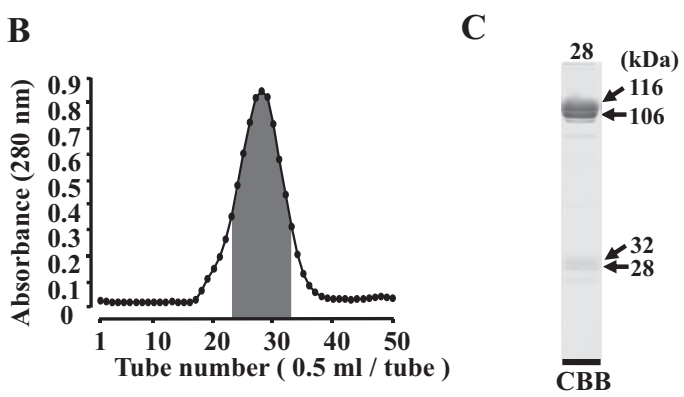
(B). The shaded area in chromatogram A indicates frac-
The (LV). The shaded area in the chromatogram $B$ indicates v. (C) $10 \%$ SDS-PAGE of the peak (fraction number 28 ) (C) $\begin{array}{llll}\text { M F YE M F YE M F YE } & \end{array}$

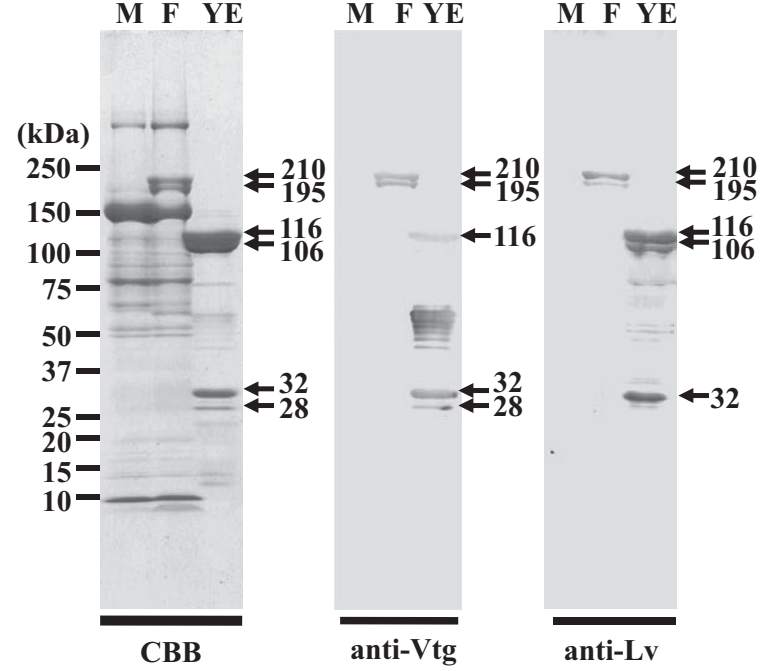

Fig. 4. 5-22.5\% SDS-PAGE and corresponding Western blots of male serum (M), female serum (F), yolk extracts (YE). Antisera against purified vitellogenin (anti-Vtg) and purified lipovitellin (antiLv) were utilized in Western blotting. CBB: Coomassie Brilliant Blue staining.

Table 1. Alignment of deduced Vtg sequences $\left(\mathrm{Vtg} 1^{\star}\right.$ and $\left.\mathrm{Vtg} 2^{\star}\right)$ with $\mathrm{N}$-terminal amino acid sequences of purified hagfish vitellogenins (210Vtg and 195Vtg) and lipovitellin heavy chains $(116 \mathrm{LvH}$ and 106LvH).

\begin{tabular}{|c|c|c|c|c|c|c|c|c|c|}
\hline Protein name & \multicolumn{9}{|c|}{$\mathrm{N}$-terminal sequence } \\
\hline Vtg1 (Amino acid position 16-23) & $\mathrm{H}$ & L & $\mathrm{E}$ & $P G$ & $\mathrm{~N}$ & $\mathrm{~T}$ & $\mathrm{Y}$ & & \\
\hline $210 \mathrm{Vtg}$ & $x$ & L & $\mathrm{E}$ & $P G$ & $\mathrm{~N}$ & $\mathrm{~T}$ & $\mathrm{Y}$ & & \\
\hline 116LvH & $\mathrm{H}$ & L & $\mathrm{E}$ & $P G$ & $\mathrm{~N}$ & $\mathrm{~T}$ & $\mathrm{Y}$ & & \\
\hline Vtg2 (Amino acid position 17-26) & $\mathrm{H}$ & $\mathrm{I}$ & Q & $R G$ & $\mathrm{~K}$ & A & $\mathrm{Y}$ & $\mathrm{L}$ & $\mathrm{Y}$ \\
\hline $195 \mathrm{Vtg}$ & $\mathrm{X}$ & I & Q & $X \mathrm{G}$ & $\mathrm{K}$ & A & $\mathrm{Y}$ & $\mathrm{L}$ & $\mathrm{Y}$ \\
\hline 106LvH & $\mathrm{H}$ & I & Q & $X G$ & $K$ & A & $Y$ & $\mathrm{~L}$ & $\mathrm{Y}$ \\
\hline
\end{tabular}

${ }^{*}$ Deduced amino acid sequences of cloned partial vtg cDNAs (vtg1; GenBank KF700373 and vtg2; GenBank KF700374)

"X": unidentified amino acid.

of $\sim 116 \mathrm{kDa}(116 \mathrm{LvH})$ and $\sim 106 \mathrm{kDa}(106 \mathrm{LvH})$. Including unidentified amino acids $(X)$, eight residues were sequenced for $210 \mathrm{Vtg}$ and $116 \mathrm{LvH}$, while 10 residues were sequenced 
for $195 \mathrm{Vtg}$ and $106 \mathrm{LvH}$ (Table 1). Moreover, these N-terminal sequences were aligned with the partial Vtg sequences which are deduced from cDNA sequences ecoding 5 '-ends of two vtg subtypes cloned in this study (vtg1; GenBank KF700373 and vtg2; GenBank KF700374). Except for the unidentified amino acids, N-termini of $210 \mathrm{Vtg}, 116 \mathrm{LvH}$ and the deduced Vtg1 were identical to each other, while $\mathrm{N}$ termini of $195 \mathrm{Vtg}, 106 \mathrm{LvH}$, and the deduced Vtg2 also appeared to be identical to each other.

\section{DISCUSSION}

Characterization of Vtg proteins and/or vtg transcripts has been pursued in various oviparous vertebrates including teleosts, amphibians and birds; only a few such studies have been performed in Agnatha, the most primitive vertebrate clade, of which hagfish is a notable representative. As described above, Yu et al. (1980) immunochemically confirmed the presence of a yolk precursor in the serum of vitellogenic female Pacific hagfish. However, characterization of the purified Vtg product remained to be completed. Therefore we initially purified Vtg of the inshore hagfish in order to conclusively characterize and identify Vtg in this species.

Detection of Vtg was carried out prior to the purification; in SDS-PAGE and Western blotting using ab. anti-YE, two female-specific YP-related polypeptides, designated as putative Vtgs (210Vtg and 195Vtg), were observed in the serum of a vitellogenic female. These polypeptides shared the typical properties of teleost Vtg, which include: (1) its specific presence in the serum of sexually mature females; (2) a common antigenicity with YP; and (3) the relatively large molecular weights $(\sim 500 \mathrm{kDa}$ to $\sim 610 \mathrm{kDa}$ in gel filtration; $168 \mathrm{kDa} \sim 240 \mathrm{kDa}$ in SDS-PAGE) (Matsubara et al., 1999; Hiramatsu et al., 2005; Sawaguchi et al., 2006; Amano et al., 2007a; Yamane et al., 2013). Following Vtg purification, the apparent molecular mass of putative hagfish Vtg was estimated as $\sim 505 \mathrm{kDa}$ by gel filtration (intact state), and this purified product dissociated into doublet bands (210Vtg and 195Vtg) in SDS-PAGE. Thus, the intact and denatured properties of the purified Vtg product matched the typical Vtg properties as described above. Namely, dimeric Vtgs may dissociate into monomeric Vtg in SDS-PAGE.

In Agnatha, Vtg has previously been purified from the blood plasma of maturing female river lampreys, using immunoadsorbent column chromatography coupled with antiserum against lamprey egg yolk (Mewes et al., 2002). Many studies have utilized anion-exchangers for the purification of teleost Vtg and succeeded to separate it from other serum proteins at high salt concentrations (reviewed by Hiramatsu et al., 2005, 2006). Anion-exchangers are also effective in separating distinct subtypes of Vtg (Hiramatsu et al., 2002c). We thus initially attempted to purify hagfish Vtg using anion-exchange chromatography. Unlike the aforementioned reports, some serum proteins other than Vtg appeared to be present in the eluted fractions (data not shown). Subsequently, we utilized a combination of the anion-exchange column and HA column. This approach could successfully separate the putative Vtgs from other serum components, although doublet putative Vtg bands (210Vtg and $195 \mathrm{Vtg}$ ) could not be separated from each other during the purification procedures in the present study.
This indicates that they represent either two closely related but distinct Vtg subtypes, or a Vtg monomer and its partial degraded (or proteolytically nicked) products. To confirm whether the purified Vtg product consists of two distinct Vtg subtypes or a mixture of proteolytic Vtg variants derived from a single $\mathrm{Vtg}$ polypeptide, N-termini of $195 \mathrm{Vtg}$ and $210 \mathrm{Vtg}$ were sequenced and aligned with amino acid sequences deduced from two distinct vtg cDNAs (vtg1 and vtg2). The results clearly confirmed that $210 \mathrm{Vtg}$ and $195 \mathrm{Vtg}$ were protein products of vtg1 and vtg2 transcripts, concluding that the purified Vtg product is a mixture of two distinct Vtg subtypes of hagfish.

Information on Vtg-related YPs in hagfish is also very limited. Lange and Richer (1981) demonstrated that a crystal structure resembling that of Lv of $X$. laevis is present in LV of Atlantic hagfish based on X-ray analysis. However, neither purification nor biochemical characterization have been yet performed for Vtg-related YPs in hagfish species.

In the present study, immunological detection of YPs was performed prior to the purification of the major Vtgderived YP products (i.e., Lvs). In SDS-PAGE of the YE, two major bands (116 LvH and 106LvH) were observed and expected to represent heavy $(H)$ chains of $L v$ on the basis of the following characteristics reported for teleost Lvs: (1) relatively large molecules ( $300 \mathrm{kDa}$ to $400 \mathrm{kDa})$, and (2) made up of at least two polypeptides, i.e., a larger heavy chain ( 94 to $\sim 110 \mathrm{kDa}$ ) and (a) smaller light chains ( 28 to $\sim 54 \mathrm{kDa}$ ), in SDS-PAGE (Hiramatsu and Hara, 1996; Matsubara et al., 1999; Hiramatsu et al., 2002a; Amano et al., 2007b). Following purification, the apparent intact mass of the purified hagfish Lv (> $669 \mathrm{kDa}$ ) was found to be much higher than those reported for fish Lvs ( 300 to $\sim 400 \mathrm{kDa})$ (Hiramatsu and Hara, 1996; Matsubara et al., 1999; Hiramatsu et al., 2002a; Amano et al., 2007b). In contrast, such a large-mass Lv (> $669 \mathrm{kDa}$ ) has also been reported for catshark Lv (Yamane et al., 2013). It is assumed that Lv of the inshore hagfish formed a large lipoprotein complex in its intact state, like assumed for Lv in the catshark. This polymerization of Lv may be related to its hydrophobicity commonly found in these species. Using SDS-PAGE, purified putative hagfish Lv dissociated into four bands ( 116, $\sim 106, \sim 32$ and $\sim 28 \mathrm{kDa}$ ); these sizes were close to those reported for $\mathrm{LvH}$ and $\mathrm{LvL}$ of teleost fishes (see above) and for those $(\sim 110$ and $\sim 30 \mathrm{kDa}$, respectively) of catshark (Yamane et al., 2013). Thus far, the YP product purified from hagfish YE in this study appeared to exhibit typical biochemical properties of Lv although its native mass was much larger than what was expected. In addition, duality of $\mathrm{LvH}$ and $\mathrm{LvL}$ subunits seems to indicate that they were derived from their two parental Vtg subtypes (see below for more details).

Purification of Vtg-related YPs has been conducted in various teleost species and the procedures typically include separations by water precipitation, ammonium sulfate precipitation, HA chromatography, gel filtration and ion exchange chromatography (Hara et al., 1993; Hiramatsu and Hara, 1996; Matsubara and Sawano, 1995; Hiramatsu et al., 2002 a, c; Fujiwara et al., 2005; Amano et al., 2007b). In the present study, it was difficult to use ion-exchange chromatography, as the putative Lv appeared to precipitate using a buffer with a low $\mathrm{NaCl}$ concentration. Yamane et al. 
(2013) purified Lv from egg yolk of the catshark by HA column eluted with $\mathrm{KP}$ buffers containing $1 \mathrm{M} \mathrm{NaCl}$. The yolk fraction containing Lv appeared to be extremely hydrophobic and was hardly dissolving into the buffer with low salt concentration. Therefore, HA chromatography was initially selected for the purification of hagfish Lv in the present study.

The precursor-product relationship between purified Vtg and YP products was initially confirmed by immunological procedures in the present study. Western blotting using antiLv specifically recognized dual Vtg polypeptides (210Vtg and $195 \mathrm{Vtg}$ ) in the serum of vitellogenic female hagfish, demonstrating that both Vtgs share their antigenicities with yolk Lv(s). This supports a general concept that these Vtgs are precursors of yolk Lv(s). Conversely, anti-Vtg recognized YPs; one (106LvH) of putative LvHs and two putative LvLs appeared to be immunoreactive to the antiserum as expected. It remains unknown why the antiserum did not react to $116 \mathrm{LvH}$ in this study. It may be that the antigenic epitopes on Vtgs are altered due to the enzymatic processing of Vtgs into Lvs in the oocytes. It is also possible that the rabbit recognized only a proportion of the highly antigenic sites of the Vtgs. Such epitopes may be present in positions other than the $\mathrm{LvH}$ domain, such as the domains of $\mathrm{LvL}$ and $\beta^{\prime}-c$ (Hiramatsu et al., 2005). A faint reaction of anti-Vtg was found even on 106 LvH and thus may support the above hypotheses.

Beside $\mathrm{LvH}$ and LvL subunits, anti-Vtg recognized multiple bands in the YE, appearing as polypeptide bands from $\sim 62$ to $\sim 43 \mathrm{kDa}$ following Western blotting. Thus, they were identified as Vtg-related yolk polypeptides. Some of these bands are expected to be peptides derived from either a part of Lv (proteolytic variants or degraded products) or other YP classes such as $\beta^{\prime}-c$ and $P v$ peptides. In fact, $\beta^{\prime}-c$ and PV domains appeared to be present in the deduced amino acid sequences of inshore hagfish vtgs (Nishimiya et al., manuscript in preparation). In this regard, some features of $\beta^{\prime}-c$ and Pv peptides should be noted here, as follows: 1) the relative masses of teleost $\beta^{\prime}$-c have been reported as being less than $\sim 20 \mathrm{kDa}$ by SDS-PAGE performed under reducing conditions (Hiramatsu and Hara, 1996; Matsubara et al., 1999; Hiramatsu et al., 2002b; Amano et al., 2007b) and 2) in teleosts, $\mathrm{Pv}$ is known to be undetectable when it is stained with CBB due to its highly phosphorylated state (Hiramatsu and Hara, 1996). As none of the above features fit to the aforementioned Vtg-related intermediate bands (bands migrating between $\sim 62$ to $\sim 43 \mathrm{kDa}$ ), they might be proteolytic variants other than typical YP classes. A possible complex peptide composed of $\beta^{\prime}-c$ and $P v$ appeared to be present in the catshark (Yamane et al., 2013), while the presence of other YP variants with variable combinations of YP domains has been found in several fishes (Sawaguchi et al., 2005; Amano et al., 2007b). Thus, fishes may differ in the mode of $\mathrm{Vtg}$ cleavage, giving rise to a variety of $\mathrm{Vtg}$ derived YP peptides.

The relationship between Vtgs and Lvs were further analyzed by the peptide sequencing in this study. Except for some unknown amino acids $(\mathrm{X}), \mathrm{N}$-terminus of $210 \mathrm{Vtg}$ was $100 \%$ identical to it of $116 \mathrm{LvH}$, as well as, it of deduced amino acid sequence of $v \operatorname{tg} 1$. On the other hand, N-terminus of $195 \mathrm{Vtg}$ peptides was $100 \%$ identical to it of $106 \mathrm{LvH}$, as well as, it of deduced amino acid sequence of vtg2. These results provide additional evidence in support of the notion that $116 \mathrm{LvH}$ and $106 \mathrm{LvH}$ are derived from the $\mathrm{N}$-terminal region of $210 \mathrm{Vtg}$ (the translation product of vtg1) and 195Vtg (vtg2), respectively.

In conclusion, purification and characterization of Vtgs and their related Lvs were performed in the inshore hagfish; the results indicated that two types of hagfish Vtg proteins (210Vtg and 195Vtg), which are products of two distinct vtg transcripts (vtg1 and $v \operatorname{tg} 2$, respectively), give rise to their derived Lvs (dual Lvs consisting of $116 \mathrm{LvH}$ and $106 \mathrm{LvH}$, respectively). These novel findings on the properties of hagfish Vtgs and YPs, as well as Vtg and Lv antisera generated in this study, will provide basic information and necessary tools to elucidate and monitor the reproductive physiology of Agnatha species, which lead us to understand the diversity and commonality of vitellogenesis in oviparous vertebrates.

\section{ACKNOWLEDGMENTS}

We are grateful to Dr. P. M. Lokman (University of Otago, New Zealand) for critical reading of the manuscript. Thanks are also due to Dr. M. Shimizu (Hokkaido University), Dr. T. Fujita (Hachinohe Institute of Technology), and Dr. H. Amano (Kitasato University) for their helpful discussions. This work was supported by JSPS KAKENHI Grant Numbers 25-1491 and 22380103.

\section{REFERENCES}

Amano H, Fujita T, Hiramatsu N, Sawaguchi S, Matsubara T, Sullivan CV, Hara A (2007a) Purification of multiple vitellogenin in grey mullet (Mugil cephalus). Mar Biol 152: 1215-1225

Amano H, Fujita T, Hiramatsu N, Shimizu M, Sawaguchi S, Matsubara $\mathrm{T}$, et al. (2007b) Egg yolk proteins in grey mullet (Mugil cephalus): purification and classification of multiple lipovitellins and other vitellogenin-derived yolk proteins. J Exp Zool 307A: 324-341

Finn RN, Kristoffersen BA (2007) Vertebrate vitellogenin gene duplication in relation to the "3R hypothesis": Correlation to the pelagic egg and the oceanic radiation of teleosts. PLOS ONE 2 (1): e169

Fujiwara Y, Fukada $\mathrm{H}$, Shimizu M, Hara A (2005) Purification of two lipovitellins and development of immunoassays for two forms of their precursors (vitellogenins) in medaka (Oryzias latipes). Gen Comp Endocrinol 143: 267-277

Hall BK (1998) Evolutionary developmental biology. 2nd ed, Chapman and Hall, London

Hall BK (1999) The neural crest in developmental and evolution. Springer Verlag, New York, pp 46-56

Hara A, Sullivan CV, Dickhoff WW (1993) Isolation and some characterization of vitellogenin and its related egg yolk proteins from coho salmon (Oncorhynchus kisutch). Zool Sci 10: 245-256

Hiramatsu N, Hara A (1996) Relationship between vitellogenin and its related egg yolk proteins in Sakhalin taimen (Hucho perryi). Comp Biochem Physiol 115A: 243-251

Hiramatsu N, Hiramatsu K, Hirano K, Hara A (2002a) Vitellogenin derived yolk proteins in a hybrid sturgeon, bester (Huso huso $x$ Acipencer ruthenus): identification, characterization and course of proteolysis during embryogenesis. Comp Biochem Physiol A Mol Inteqr Physiol 131: 429-441

Hiramatsu N, Matsubara T, Weber GM, Sullivan CV, Hara A (2002b) Vitellogenesis in aquatic animals. Fish Sci 68 (Suppl I): 694699

Hiramatsu N, Hara A, Hiramatsu K, Fukada H, Weber GM, Denslow ND, Sullivan CV (2002c) Vitellogenin-derived yolk proteins of white perch, Morone americana: purification, characterization and vitellogenin-receptor binding. Biol Reprod 67: 655-667 
Hiramatsu N, Cheek AO, Sullivan CV, Matsubara T, Hara A (2005) Vitellogenesis and endocrine disruption. In Biochemistry and molecular biology of fishes, vol 6 . Ed by Mommsen TP, Moon TW, Elsevier, Amsterdam, 562 pp 431-471

Hiramatsu N, Matsubara T, Fujita T, Sullivan CV, Hara A (2006) Multiple piscine vitellogenins: biomarkers of fish exposure to estrogenic endocrine disruptors in aquatic environments. Mar Biol 149: 35-47

Lange RH, Richer HP (1981) A symmetric lipovitellin-phosvitin dimmer in cyclostome yolk platelet crystals: Structural and biochemical observations. J Mol Biol 148: 487-491

Matsubara T, Sawano K (1995) Proteolytic cleavage of vitellogenin and yolk proteins during vitellogenin uptake and oocyte maturation in barfin flounder (Verasper moseri). J Exp Zool 272: 34-45

Matsubara T, Ohkubo N, Andoh T, Sullivan CV, Hara A (1999) Two forms of vitellogenin, yielding two distinct lipovitellins, play different roles during oocytematuration and early development of barfin flounder, Verasper moseri, a marine teleost that spawns pelagic eggs. Dev Biol 213: 18-20

Meininger T, Raag R, Roderick S, Banaszak LJ (1984) Preparation of single crystals of a yolk lipoprotein. J Mol Biol 179: 759-764

Mewes KR, Latz M, Golla H, Fischer A (2002) Vitellogenin from female estradiol-stimulated male river lampreys (Lampetra fluviatilis L.). J Exp Zool 292: 52-72

Ota KG, Kuratani S (2006) The history of scientific endeavors towards understanding hagfish embryology. Zool Sci 23: 403418

Raag R, Appelt K, Xuong NH, Banaszak LJ (1988) Structure of the lamprey yolk lipid-protein complex lipovitellin-phosvitin at $2.8 \AA$ resolution. J Mol Biol 200: 553-569

Reading B, Hiramatsu N, Sawaguchi S, Matsubara T, Hara A, Lively M, Sullivan CV (2009) Conserved and variant molecular and functional features of multiple egg yolk precursor proteins (vitellogenins) in white perch (Morone americana) and other teleosts. Mar Biotechnol 11: 169-187
Sawaguchi S, Koya Y, Yoshizaki N, Ohkubo N, Andoh T, Hiramatsu $\mathrm{N}$, et al. (2005) Multiple vitellogenins (Vtgs) in mosquitofish (Gambusia affinis): identification and characterization of three functional Vtg genes and their circulating and yolk proteins products. Biol Reprod 72: 1045-1060

Sawaguchi S, Kagawa H, Ohkubo N, Hiramatsu N, Sullivan CV, Matsubara T (2006) Molecular characterization of three forms of vitellogenin and their yolk protein products during oocyte growth and maturation in red seabream (Pagrus major), a marine teleost spawning pelagic eggs. Mol Reprod Dev 73: 719-736

Sharrock WJ, Rosenwasser TA, Gould J, Knott J, Hussey D, Gordon JI, Banaszak LJ (1992) Sequence of lamprey vitellogenin. Implications for the lipovitellin crystal structure. J Mol Biol 226: 903907

Specker JL, Sullivan CV (1994) Vitellogenesis in fishes: status and perspectives. In "Perspectives in Comparative Endocrinology" Ed by KG Davey, RE Peter, SS Tobe, National Research Council, Ottwa, pp 304-315

Wallace RA (1985) Vitellogenesis and oocyte growth in nonmammalian vertebrates. In "Developmental Biology, Vol 1" Ed by LW Browder, Plenum Press, New York, pp 127-177

Yamane K, Yagai T, Nishimiya O, Sugawara R, Amano H, Fujita T, et al. (2013) Characterization of vitellogenin and its derived yolk proteins in cloudy catshark (Scyliorhinus torazame). Fish Physiol Biochem 2: 373-390

Yu JLY, Dickhoff WW, Inui Y, Gorbman A (1980) Sexual patterns of protein metabolism in liver and plasma of hagfish, Eptatretus stouti with special reference to vitellogenesis. Comp Biochem Physiol B Biochem physiol 65: 111-117

Yu JLY, Dickhoff WW, Swanson P, Gorbman A (1981) Vitellogenesis and its hormonal regulation in the pacific hagfish, Eptatretus stouti L. Gen Comp Endocrinol 43: 492-502

(Received November 13, 2013 / Accepted December 13, 2013) 